Cahiers $d u$ MONDE RUSSE

\section{Cahiers du monde russe}

Russie - Empire russe - Union soviétique et États indépendants

$43 / 4 \mid 2002$

Intellectuels et intelligentsia

\title{
Douglas R. Weiner, A little corner of freedom
}

\section{Marie-Hélène Mandrillon}

\section{OpenEdition \\ Journals}

Édition électronique

URL : https://journals.openedition.org/monderusse/4041

DOI : 10.4000/monderusse.4041

ISSN : $1777-5388$

Éditeur

Éditions de l'EHESS

Édition imprimée

Date de publication : 30 décembre 2002

Pagination : 753-756

ISBN : 2-7132-1796-2

ISSN : $1252-6576$

Référence électronique

Marie-Hélène Mandrillon, « Douglas R. Weiner, A little corner of freedom », Cahiers du monde russe [En ligne], 43/4 | 2002, mis en ligne le 17 juin 2009, consulté le 03 septembre 2022. URL : http:// journals.openedition.org/monderusse/4041 ; DOI : https://doi.org/10.4000/monderusse.4041

Ce document a été généré automatiquement le 3 septembre 2022

Tous droits réservés 


\title{
Douglas R. Weiner, A little corner of freedom
}

\author{
Marie-Hélène Mandrillon
}

\section{RÉFÉRENCE}

Douglas R. WEINER, A little corner of freedom. Russian nature protection from

Stalin to Gorbachev. Berkeley, University of California Press, 1999, 556 p.

1 Cet ouvrage de Douglas Weiner constitue en quelque sorte la prolongation du précédent, Models of nature: ecology, conservation, and cultural revolution in Soviet Russia ${ }^{1}$. Avec cette nouvelle somme, Douglas Weiner s'affirme comme l'historien de la «naučnaja obščstvennost'", confirmant que la portée de son travail s'étend bien au-delà de l'activisme environnemental. Si le premier volume était consacré essentiellement aux années 1920 et au début des années 1930, celui-ci embrasse l'ensemble de la période stalinienne et se poursuit jusqu'à la fin de l'URSS. Autre différence entre les deux publications, le périmètre des sources mobilisées s'est considérablement accru, ouverture des archives oblige. Mais il serait infondé de dire que Douglas Weiner en a bénéficié opportunément car son rôle dans l'entreprise d'ouverture et d'exploitation des fonds d'archives publics soviétiques est celui d'un artisan actif, comme en témoigne par exemple sa participation à la publication du recueil de documents Ekologija $i$ vlast', 1917-1990, paru à Moscou en 1999 dans la série Rossija. XX vek.

Dans ce nouveau livre, l'auteur suit du début à la fin du régime soviétique les vicissitudes d'un petit groupe de scientifiques, biologistes pour l'essentiel, botanistes, zoologistes, podologues et géographes de terrain, qui constituent en 1924 la VOOP (Vserossijskoe obščestvo ohrany prirody), la Société panrusse de protection de la nature, comme héritière des sociétés savantes d'Ancien Régime avec ses règles démocratiques de fonctionnement interne, son atmosphère de club élitiste, et se donnant pour objectif essentiel la préservation et l'extension du réseau des zapovedniki, sorte de parcs nationaux, de réserves inviolables, destinés à servir d'étalon d'une nature "vierge ». 
Qualifiée de "réserve pour l'espèce en voie de disparition des scientifiques bourgeois ", la VOOP compte, en 1932, 17000 membres qui assurent l'administration d'un réseau de 70 zapovedniki répartis sur tout le territoire de l'Union soviétique et représentant une superficie de plus de six millions d'hectares. Weiner s'interroge sur les raisons du succès de cette entreprise qui voit ce groupe « indépendant et critique » survivre au pouvoir de Stalin et de ses successeurs, mais aussi sur les raisons de son échec, à la fois scientifique et politique, à savoir son incapacité à peser sur la définition d'une politique de protection de l'environnement en URSS.

3 La démarche de Weiner est stimulante car, ayant défini la naučnaja ob̌̌čestvennost' comme une catégorie sociale fondée sur des compétences professionnelles, un esprit de corps, un credo scientifique, des valeurs morales et notamment un sens du devoir vis-àvis de la Science, et en conséquence du service de l'État, il ne s'épuise pas à trouver une réponse qui soit unique et ultime, mais échafaude des faisceaux d'explications, s'appuyant sur des sources recoupées, ou d'hypothèses dans les cas où ni les archives disponibles, ni les témoignages recueillis a posteriori ne parviennent à emporter sa conviction.

4 À travers la description des " guerres des zapovedniki » qui se succèdent des années 1920 jusqu'aux années 1950, Weiner analyse les différentes stratégies de survie de ce groupe vis-à-vis du régime. Premier constat : même durant la période la plus aiguë de la terreur stalinienne, on déplore relativement peu de victimes parmi ces activistes. C'est dû essentiellement au fait que ces nombreux espaces de nature protégée, gérés directement par des scientifiques, souvent situés dans des régions périphériques éloignées et difficiles d'accès, ont concrètement servi de refuge physique aux victimes de purges et de persécutions, leur évitant souvent l'arrestation et la mort. Néanmoins, la survie du groupe et la défense des zapovedniki apparaît comme une "marche sur la corde raide». Quatre facteurs interviennent, qui se révèlent déterminants en fonction des périodes et des situations, et dont il apparaît impossible de déterminer précisément la part de chacun, vu leur étroite imbrication.

Le premier facteur est la stratégie classique du patronage : il est clair que le réseau des zapovedniki n'aurait pu se maintenir sans la protection de responsables politiques locaux ou régionaux. Les motivations des parrains sont diverses - disposer sur son territoire d'une réserve scientifique peut être prestigieux, rapporter en termes d'allocations budgétaires, aider à la construction d'une voie de communication, etc. Chez certains cadres intermédiaires, et en particulier les secrétaires d'obkom, intervient un réflexe de satrape qui consiste à protéger « ses » choses et « ses » gens. L'ouvrage apporte ce faisant un nouvel éclairage sur certains épisodes de conflits entre les autorités de la RSFSR et le pouvoir fédéral, notamment en 1951. Chez les dirigeants de l'Académie des sciences, on trouve des sympathisants à la cause, dont le président Nesmejanov, au début des années 1950 ; et, parmi les administrateurs du Gosplan, des personnalités en position d'infléchir la portée d'une décision politique, sous Stalin comme sous Hruščev. Pour les dirigeants du komsomol dans les années 1960, parrainer les groupes d'étudiants, les družiny, présente le double avantage de fournir une image un peu attractive tout en exerçant un contrôle.

Un autre type de protection provient de l'adhésion, proclamée ou authentique, de la part des dirigeants de la VOOP aux objectifs du régime. Ce mimétisme ou cette stratégie du caméléon repose sur des valeurs partagées : une même opposition à la propriété privée, aux forces du marché, une même croyance qu'un État fort et centralisé est mieux à même de protéger la nature. Ceci permet à la VOOP d'inscrire officiellement son action dans la 
« construction du socialisme», fût-ce au prix de concessions mineures ou de façade. L'opposition porte sur le mode de développement tel qu'il est mis en œuvre à partir du $\mathrm{I}^{\mathrm{er}}$ Plan quinquennal et, par la suite, sur l'absence d'association des scientifiques à l'élaboration des politiques publiques, ce qui les conduira à se figer dans une attitude d'attente vaine et à fustiger l'ignorance des décideurs et la grossièreté des exécutants. Et, de fait, Weiner établit qu'à l'origine de chacune des guerres successives engagées par le pouvoir contre les zapovedniki ou leurs défenseurs, on retrouve une situation de concurrence, de conflits d'usage portant sur les ressources naturelles ou l'occupation des sols.

7 Une autre manifestation de "patriotisme soviétique ", de la part des militants de la protection de la nature, réside dans l'utilisation massive de l'arme de la « honte », c'està-dire dans l'étalage qu'ils font auprès des responsables politiques de l'humiliation qu'ils ressentent à devoir reconnaître devant leurs collègues étrangers les retards ou les erreurs de la politique de leur pays. Weiner souligne la difficulté à faire définitivement en la matière la part de la ruse et de la conviction, en dépit des sources d'archives et des témoignages rétrospectifs.

Dernier élément de la stratégie de groupe - ou individuelle - qui assure la pérennité de la VOOP : l'image soigneusement cultivée que les activistes donnent d'eux-mêmes, celle de marginaux, d'excentriques, d'originaux. Hruščev les qualifiera de « @udaki» et aucun dirigeant ne considérera qu'une quelconque opposition sérieuse à son pouvoir puisse émaner de tels personnages. Là encore, cette attention portée à l'image donnée vis-à-vis du pouvoir est une réponse à la contrainte exercée par un environnement hostile mais elle renvoie également à un trait constitutif de l'identité du mouvement.

Ainsi, dans l'immédiat après-guerre, la VOOP élargit son recrutement au-delà des cercles académiques vers les enseignants et les scolaires. Elle reçoit également, nolens volens, les adhésions de "membres juridiques", c'est-à-dire d'entreprises, d'administrations entières, pour atteindre 100000 adhérents au début des années 1950. Dès lors, cette organisation, commençant à devenir « de masse », attire l'attention du pouvoir qui va chercher à en contrôler la direction, ce qui sera réalisé entre 1953 et 1955. La «vieille garde» abandonnera alors cette "courroie de transmission», laissant la nouvelle organisation gonfler sans elle jusqu'à 29 millions de membres au début des années 1980, pour trouver refuge dans une autre société amie, la MOIP (Moskovskoe obščestvo ispytatelej prirody), la Société des naturalistes de Moscou, où les critères professionnels, l'esprit de club et les règles de fonctionnement sont préservés.

10 Même s'ils constituent la trame de son ouvrage, Weiner ne limite pas son exploration aux conflits avec le pouvoir. Il mesure aussi la capacité des " professeurs bourgeois » à transmettre leur credo scientifique et leur éthique professionnelle à la génération des étudiants des années 1960 dont ils inspirent l'activisme au sein des družiny. On suit parfaitement le passage qui s'effectue alors d'une attitude de fétichisation de la nature, qui présidait au principe de l'inviolabilité des zapovedniki, à une approche en termes de protection de la biodiversité, qui se trouve être celle que partage alors la communauté internationale. On voit apparaître parmi ces étudiants les futurs acteurs des mouvements écologiques qui se développeront pendant la perestroïka ainsi que les fondateurs des institutions et des politiques de l'environnement sous Gorbačev et El'cin.

11 À la même période, le corporatisme des défenseurs de la nature se trouve également confronté à la question des alliances avec des scientifiques de disciplines ou d'horizons différents. Notamment avec ceux qui se regrouperont à Akadem-gorodok - la cité 
scientifique de Novossibirsk à partir de 1957 - ainsi qu'avec d'autres groupes sociaux (ingénieurs, administrateurs, chercheurs en sciences humaines et sociales, écrivains, journalistes) dont l'engagement en faveur de la protection de l'environnement a d'autres motivations et d'autres causes. L'auteur montre bien le mépris « de caste» envers les vydvižency et l'arrogance toute scientiste à l'encontre des non-scientifiques qui compromettront bien des entreprises.

12 Au final, le lecteur trouvera une magistrale préhistoire de l'écologie en Russie, conjuguée avec l'histoire très concrète de la traversée du siècle par un groupe professionnel. Juste un « archipel de liberté ».

\section{NOTES}

1. Bloomington-Indianapolis, Indiana University Press, 1988, 312 p. Cf. mon compte rendu in Annales ESC, 2, 1989, p. 304. 